\title{
Piecewise Linear Model-Based Image Enhancement
}

\author{
Fabrizio Russo \\ Department of Electrical, Electronic and Computer Engineering (DEEI), University of Trieste, \\ Via Valerio 10, Trieste 34127, Italy \\ Email:rusfab@univ.trieste.it
}

Received 1 September 2003; Revised 23 March 2004

\begin{abstract}
A novel technique for the sharpening of noisy images is presented. The proposed enhancement system adopts a simple piecewise linear (PWL) function in order to sharpen the image edges and to reduce the noise. Such effects can easily be controlled by varying two parameters only. The noise sensitivity of the operator is further decreased by means of an additional filtering step, which resorts to a nonlinear model too. Results of computer simulations show that the proposed sharpening system is simple and effective. The application of the method to contrast enhancement of color images is also discussed.
\end{abstract}

Keywords and phrases: image enhancement, sharpening, noise reduction, nonlinear filters.

\section{INTRODUCTION}

It is known that a critical issue in the enhancement of images is the noise increase that is typically produced by the sharpening process [1]. A classical example is represented by the linear unsharp masking (UM) method. Since a fraction of the high-pass filtered image is added to the original data, the resulting effect produces edge enhancement and noise amplification as well. In order to address this issue, more effective approaches resort to nonlinear filtering that can realize a better compromise between image sharpening and noise attenuation $[2,3,4,5,6]$. In particular, weighted medians (WMs) have been successfully experimented as a replacement for high-pass linear filters in the UM scheme [7]. In this framework, methods based on permutation weighted medians (PWMs) offer very interesting results because they can prevent the noise amplification during the enhancement process $[8,9]$. Polynomial UM approaches constitute another family of nonlinear methods for image enhancement. Interesting examples include the Teager-based operator $[10,11]$ and the cubic UM technique [12]. Rational UM [13] represents a powerful approach to contrast enhancement. It can avoid noise amplification and excessive overshoot on sharp details. Nonlinear methods based on fuzzy models have also been investigated. Indeed, fuzzy systems are well suited to model the uncertainty that occurs when conflicting operations should be performed, for example, detail sharpening and noise cancellation $[14,15,16]$. The most effective approaches can enhance the image data without increasing the noise. However, their ability to reduce the noise during the sharpening process is limited. In this respect, methods based on forward and backward (FAB) anisotropic diffusion constitute a powerful class of enhancement techniques $[17,18]$. Since anisotropic diffusion is typically an iterative process, the noise can be progressively reduced by means of an appropriate choice of parameter settings.

In this paper, a new simple technique for the enhancement of noisy images is presented. The proposed method improves our previous approach [19] from the point of view of architectural complexity and control of the nonlinear behavior. The new algorithm adopts only one piecewise linear (PWL) function to combine the smoothing and sharpening effects. A two-pass implementation of the method is also presented. As a result, noise reduction and edge enhancement can be achieved. This paper is organized as follows. Section 2 introduces a simple PWL model for image enhancement, Section 3 describes the complete two-pass enhancement architecture, Section 4 shows results of computer simulations, Section 5 addresses parameter tuning, Section 6 presents an application to color image processing, and finally, Section 7 reports conclusions.

\section{A SIMPLE PWL MODEL FOR IMAGE ENHANCEMENT}

We suppose that we deal with digitized images having $L$ gray levels. Let $x(\mathbf{n})$ be the pixel luminance at location $\mathbf{n}=\left[n_{1}, n_{2}\right]$ in the input image. The enhancement algorithm operates on a $3 \times 3$ window around $x(\mathbf{n})$. Let $x_{1}(\mathbf{n}), x_{2}(\mathbf{n}), \ldots, x_{N}(\mathbf{n})$ briefly denote the group of $N=8$ neighboring pixels, as shown in Figure $1(0 \leq x(\mathbf{n}) \leq L-1$; $\left.0 \leq x_{i}(\mathbf{n}) \leq L-1, i=1, \ldots, 8\right)$. 


\begin{tabular}{|l|l|l|}
\hline$x_{1}(\mathbf{n})$ & $x_{2}(\mathbf{n})$ & $x_{3}(\mathbf{n})$ \\
\hline$x_{4}(\mathbf{n})$ & $x(\mathbf{n})$ & $x_{5}(\mathbf{n})$ \\
\hline$x_{6}(\mathbf{n})$ & $x_{7}(\mathbf{n})$ & $x_{8}(\mathbf{n})$ \\
\hline
\end{tabular}

Figure 1: $3 \times 3$ window.

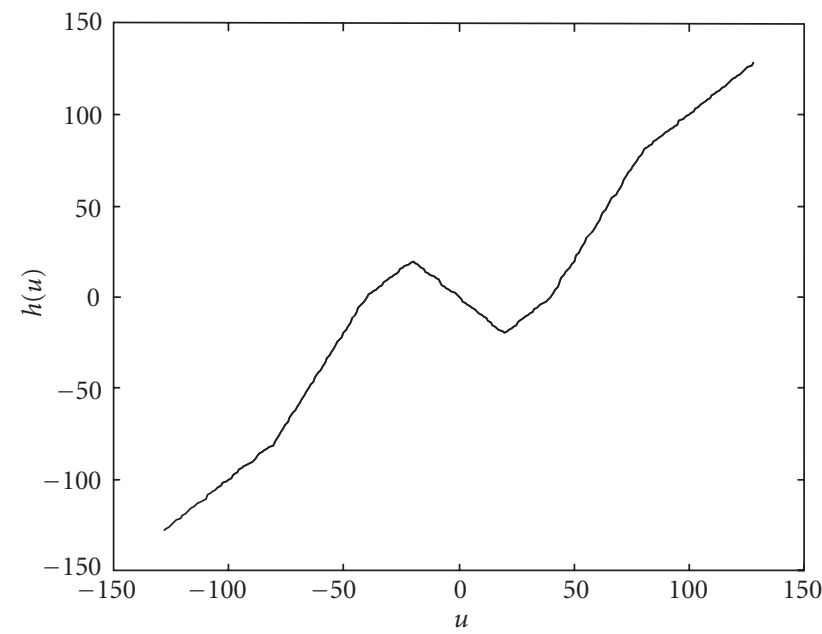

FigURE 2: Example of graphical representation of function $h(u)$.

Let $y(\mathbf{n})$ represent the output of the enhancement system. The algorithm is described by the following relationships:

$$
\begin{gathered}
y(\mathbf{n})=x(\mathbf{n}) \oplus s(\mathbf{n}), \\
s(\mathbf{n})=\frac{1}{N} \sum_{i=1}^{N} h\left(\Delta x_{i}(\mathbf{n})\right), \\
\Delta x_{i}(\mathbf{n})=x(\mathbf{n})-x_{i}(\mathbf{n}),
\end{gathered}
$$

where symbol $\oplus$ represents the bounded sum $a \oplus b=\min \{a+$ $b, L-1\}$ and $h$ is a PWL function whose behavior is controlled by two parameters $k_{\mathrm{sm}}$ and $k_{\mathrm{sh}}$ :

$$
h(u)= \begin{cases}\frac{1}{2} k_{\mathrm{sh}} u, & u<-4 k_{\mathrm{sm}}, \\ k_{\mathrm{sh}}\left(u+2 k_{\mathrm{sm}}\right), & -4 k_{\mathrm{sm}} \leq u<-2 k_{\mathrm{sm}}, \\ u+2 k_{\mathrm{sm}}, & -2 k_{\mathrm{sm}} \leq u<-k_{\mathrm{sm}}, \\ -u, & -k_{\mathrm{sm}} \leq u<k_{\mathrm{sm}}, \\ u-2 k_{\mathrm{sm}}, & k_{\mathrm{sm}} \leq u<2 k_{\mathrm{sm}}, \\ k_{\mathrm{sh}}\left(u-2 k_{\mathrm{sm}}\right), & 2 k_{\mathrm{sm}} \leq u<4 k_{\mathrm{sm}}, \\ \frac{1}{2} k_{\mathrm{sh}} u, & u \geq 4 k_{\mathrm{sm}} .\end{cases}
$$

An example of graphical representation of $h(u)$ is depicted in Figure $2\left(k_{\mathrm{sm}}=20\right.$ and $\left.k_{\mathrm{sh}}=2\right)$.

The basic idea is very simple. It takes into account the luminance differences $\Delta x_{i}$ between the central pixel and its neighbors (see (3)). When these differences are small, the method performs smoothing, that is, an action that aims at reducing such differences in the enhanced image. Conversely, when the luminance differences are high, sharpening is provided, that is, an effect that tends to increase such differences. According to (4), as $\left|\Delta x_{i}\right|$ increases, its effect in (2) becomes quite different. More precisely, this effect is strong smoothing for very small differences $\left(\left|\Delta x_{i}(\mathbf{n})\right|<k_{\text {sm }}\right)$, weak smoothing for small differences $\left(k_{\mathrm{sm}} \leq\left|\Delta x_{i}(\mathbf{n})\right|<2 k_{\mathrm{sm}}\right)$, strong sharpening for medium differences $\left(2 k_{\mathrm{sm}} \leq\left|\Delta x_{i}(\mathbf{n})\right|<4 k_{\mathrm{sm}}\right)$, and weak sharpening for large differences $\left(\left|\Delta x_{i}(\mathbf{n})\right| \geq 4 k_{\mathrm{sm}}\right)$. The shape of $h(u)$ has been designed to gradually combine the smoothing and sharpening effects. The choice of a 7-segment model is based on experimentation. It is a compromise between complexity and effectiveness. Models with more segments require more parameters and do not yield a significant improvement. On the other hand, models with less segments do no provide enough performance and flexibility.

In our model, the actual amount of smoothing and sharpening can be controlled by the parameters $k_{\mathrm{sm}}$ and $k_{\mathrm{sh}}$, respectively. When $k_{\mathrm{sh}}=0$, no sharpening is performed and the resulting action is smoothing only. Thus (4) becomes

$$
h(u)= \begin{cases}0, & u<-2 k_{\mathrm{sm}}, \\ u+2 k_{\mathrm{sm}}, & -2 k_{\mathrm{sm}} \leq u<-k_{\mathrm{sm}}, \\ -u, & -k_{\mathrm{sm}} \leq u<k_{\mathrm{sm}}, \\ u-2 k_{\mathrm{sm}}, & k_{\mathrm{sm}} \leq u<2 k_{\mathrm{sm}}, \\ 0, & u \geq 2 k_{\mathrm{sm}} .\end{cases}
$$

When $\left|\Delta x_{i}(\mathbf{n})\right|<k_{\mathrm{sm}}(i=1,2, \ldots, N)$, the luminances of the neighboring pixels are close to the value of the central element and we have $h\left(\Delta x_{i}(\mathbf{n})\right)=-\Delta x_{i}(\mathbf{n})$. Thus, according to (1) and (2), the filter realizes the arithmetic mean of the pixel luminances in the neighborhood and the resulting effect is a strong smoothing action:

$$
y(\mathbf{n})=\frac{1}{N} \sum_{i=1}^{N} x_{i}(\mathbf{n})
$$

The filtering process aims at excluding luminance values $x_{i}(\mathbf{n})$ that are very different from $x(\mathbf{n})$ in order to avoid blurring the image details. According to this rule, when $\left|\Delta x_{i}(\mathbf{n})\right| \geq 2 k_{\text {sm }}$, we have $h\left(\Delta x_{i}(\mathbf{n})\right)=0$. A gradual transition between $h\left(\Delta x_{i}(\mathbf{n})\right)=-\Delta x_{i}(\mathbf{n})$ and $h\left(\Delta x_{i}(\mathbf{n})\right)=0$ is provided when $k_{\mathrm{sm}} \leq\left|\Delta x_{i}(\mathbf{n})\right|<2 k_{\mathrm{sm}}$ (see (5)). As abovementioned, the smoothing behavior is controlled by the parameter $k_{\mathrm{sm}}$. Large values of $k_{\mathrm{sm}}$ increase the noise cancellation, while small values increase the detail preservation. Notice that smoothing requires that $h\left(\Delta x_{i}(\mathbf{n})\right)<0$ when $\Delta x_{i}(\mathbf{n})>0$ and $h\left(\Delta x_{i}(\mathbf{n})\right)>0$ when $\Delta x_{i}(\mathbf{n})<0$.

Now, we introduce the sharpening action. If we choose $k_{\text {sh }}>0$ (typically $k_{\text {sh }} \leq 6$ ), a sharpening effect is applied to the image pixels when $\left|\Delta x_{i}(\mathbf{n})\right|>2 k_{\text {sm }}$ (see (4)). Since sharpening can be considered as the opposite of the smoothing action [14,15], we set $h\left(\Delta x_{i}(\mathbf{n})\right)>0$ when $\Delta x_{i}(\mathbf{n})>2 k_{\text {sm }}$ and $h\left(\Delta x_{i}(\mathbf{n})\right)<0$ when $\Delta x_{i}(\mathbf{n})<-2 k_{\mathrm{sm}}$. In particular, this sharpening effect is stronger if $2 k_{\mathrm{sm}} \leq\left|\Delta x_{i}(\mathbf{n})\right|<4 k_{\mathrm{sm}}$ and weaker when $\left|\Delta x_{i}(\mathbf{n})\right| \geq 4 k_{\text {sm }}$ (look at the difference in the 
slope of the graph in Figure 2). This choice aims at avoiding an annoying excess of sharpening along the object contours of the image.

\section{IMPROVING THE ENHANCEMENT PROCESS}

The quality of the enhanced image can be improved by introducing a further processing step for the cancellation of possible outliers still remaining in the image. If the image is corrupted by Gaussian noise, these outliers typically represent the fraction of noise located on the "tail" of the Gaussian distribution. Even if the probability of occurrence of these outliers is low, their presence can be rather annoying, especially in the uniform regions of the image. The processing scheme described by (1), (2), (3), and (4) would require a large value of $k_{\mathrm{sm}}$ to smooth out this kind of noise and, as a consequence, some blurring of fine details could be produced. A more suitable choice is the adoption of an additional filtering step devoted to the cancellation of these outliers. This choice permits us to use a smaller value of $k_{\mathrm{sm}}$ that can satisfactorily preserve the image details. The filter for outlier removal adopts a different approach to process the luminance differences in the window. Indeed, the filter aims at detecting pixel luminances that are very different from those of the neighborhood. The filter is defined by the following relationship:

$y(\mathbf{n})=x(\mathbf{n})-\underset{i=1,2, \ldots, N}{\operatorname{MIN}}\left\{g\left(\Delta x_{i}(\mathbf{n})\right)\right\}+\underset{i=1,2, \ldots, N}{\operatorname{MIN}}\left\{g\left(-\Delta x_{i}(\mathbf{n})\right)\right\}$,

where $g$ is a nonlinear function:

$$
g(v)= \begin{cases}v, & 0<v \leq L-1 \\ 0, & v \leq 0\end{cases}
$$

The shape of function $g$ is chosen to achieve the exact correction in the ideal case of an outlier in a uniform neighborhood. As an example, let $x(\mathbf{n})=a$ be a positive outlier and let $x_{i}(\mathbf{n})=b(i=1,2, \ldots, N)$ be the luminance values of the neighboring pixels $(a>b)$. Since $\Delta x_{i}(\mathbf{n})=a-b>0$, we have $g\left(\Delta x_{i}(\mathbf{n})\right)=a-b$ and $g\left(-\Delta x_{i}(\mathbf{n})\right)=0$. Thus (7) yields the exact value $y(\mathbf{n})=b$. The filtering action defined by (7) and (8) can be applied after the sharpening process in order to remove outliers. A better choice, however, is to apply this filtering to the noisy input data before the enhancement process, thus avoiding amplification of these outliers. The influence of the different parameter settings and processing strategies can be highlighted by some application examples. Figure 3a shows a synthetic test image and Figure $3 \mathrm{~b}$ the same picture corrupted by Gaussian noise with variance 50 . The result of the application of our method $\left(k_{\mathrm{sm}}=10, k_{\mathrm{sh}}=5\right)$ without additional processing is reported in Figure $3 c$. The presence of many outliers is apparent. A larger value of $k_{\mathrm{sm}}$ can smooth out this noise as shown in Figure $3 \mathrm{~d}\left(k_{\mathrm{sm}}=20\right.$, $k_{\mathrm{sh}}=5$ ). If fine details were present in the image, however, this choice would produce some blurring. The result yielded by the improved enhancement process adopting additional filtering are depicted in Figure $3 \mathrm{e}$ (postfiltering, $k_{\mathrm{sm}}=15$,

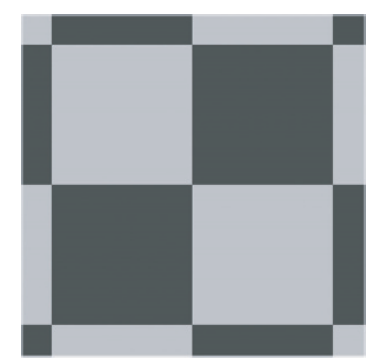

(a)

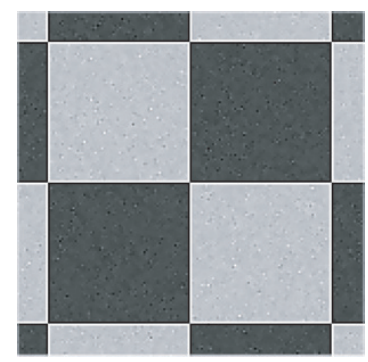

(c)

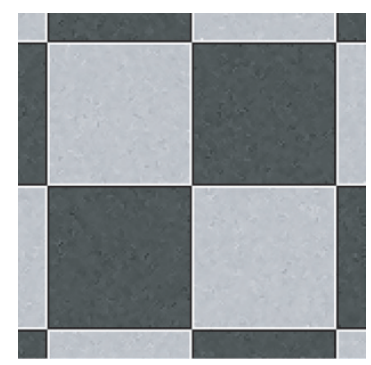

(e)

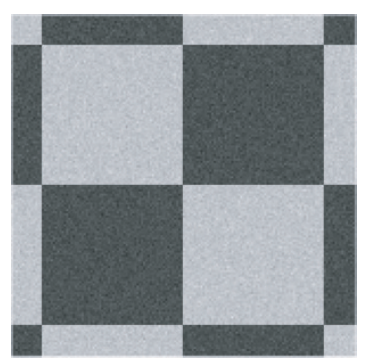

(b)

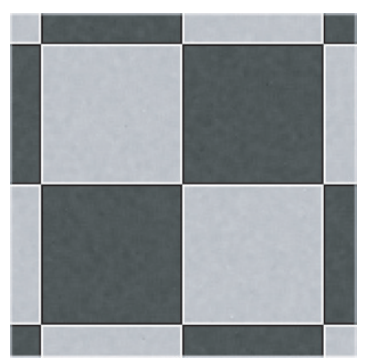

(d)

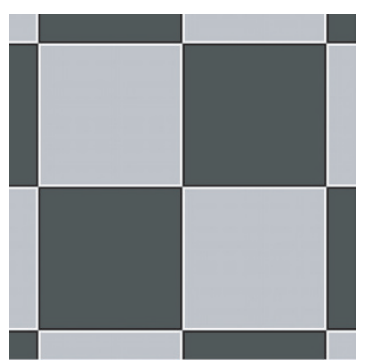

(f)
Figure 3: Details of (a) a synthetic image, (b) image corrupted by Gaussian noise with variance 50 , (c) enhanced image $\left(k_{\mathrm{sm}}=10\right.$, $k_{\mathrm{sh}}=5$, no additional processing), (d) enhanced image $\left(k_{\mathrm{sm}}=20\right.$, $k_{\mathrm{sh}}=5$, no additional processing), (e) enhanced image $\left(k_{\mathrm{sm}}=15\right.$, $k_{\mathrm{sh}}=5$, postprocessing), and (f) enhanced image $\left(k_{\mathrm{sm}}=15, k_{\mathrm{sh}}=\right.$ 5 , preprocessing).

$k_{\mathrm{sh}}=5$ ) and Figure 3f (prefiltering, $k_{\mathrm{sm}}=15, k_{\mathrm{sh}}=5$ ). We can observe that the latter gives the best result. As above mentioned, the smoothing action can easily be controlled by varying the value of $k_{\mathrm{sm}}$. A suitable choice can realize a compromise between noise cancellation and preservation of fine details and textures.

\section{RESULTS}

We performed many computer simulations in order to validate the proposed enhancement technique. In this experiment, we considered the $512 \times 512$ "Tiffany" picture (Figure $4 \mathrm{a}$ ) and we generated a noisy image by adding zeromean Gaussian noise with variance 50 (Figure 4b). The result yielded by the classical linear UM scheme is depicted in 


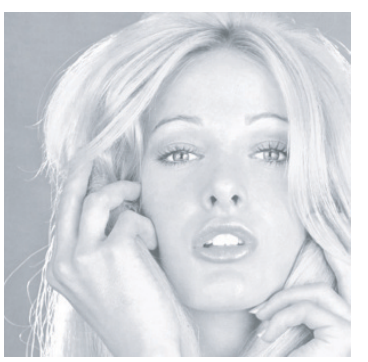

(a)

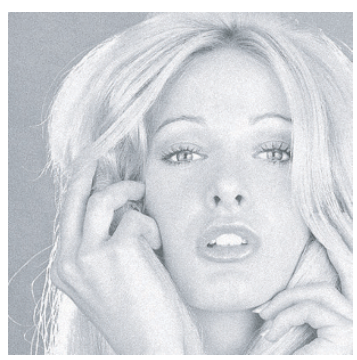

(c)

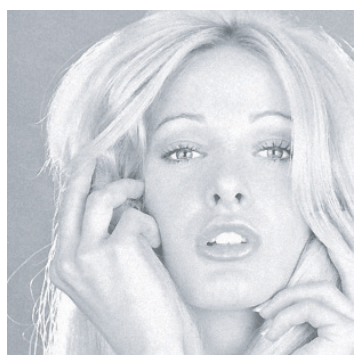

(e)

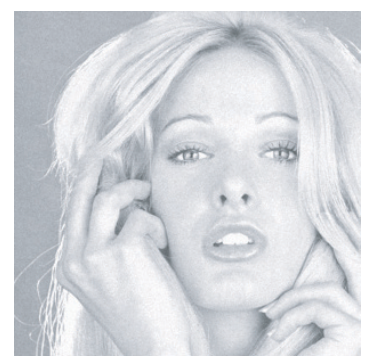

(b)

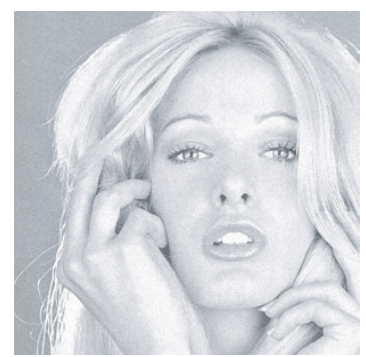

(d)

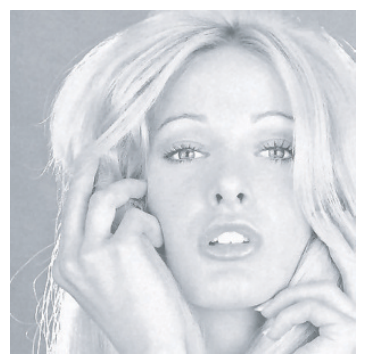

(f)

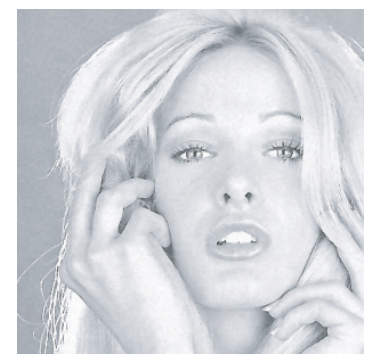

(g)

FIgURe 4: (a) Original image, (b) noisy image, (c) results given by linear UM, (d) WM UM, (e) PWM-UM, (f) FAB anisotropic diffusion, and $(\mathrm{g})$ proposed method.

Figure $4 \mathrm{c}$. (We set $\lambda=0.4$, where $\lambda$ is the tuning parameter that defines the amount of sharpening.) We can observe that the noise increased significantly as an effect of the sharpening action and the result is very annoying. A better result is offered by the nonlinear UM based on the WM (Figure 4d, $\lambda=0.5)$. However, its sensitivity to noise is rather high. Nonlinear UM based on PWMs represents a much powerful choice (Figure 4e). We considered the algorithm that allows thresholding $(L=2, \lambda=0.8, T=50)$ [9]. Observing the image in Figure $4 \mathrm{e}$, basically, no noise amplification is perceivable with respect to the input data.

An excellent combination of smoothing and sharpening is given by $\mathrm{FAB}$ anisotropic diffusion. We chose the algorithm that adopts the Gaussian-shaped function for the conduction coefficient and the following parameter settings: $\beta_{1}(1)=50$, $\beta_{2}(1)=300, \gamma=0.5$, and number of passes $=3$ [18]. The corresponding result is shown in Figure $4 \mathrm{f}$. Finally, the image yielded by our technique adopting preprocessing is represented in Figure $4 \mathrm{~g}\left(k_{\mathrm{sh}}=5, k_{\mathrm{sm}}=15\right)$. The good performance in reducing noise is apparent. The processed picture looks almost noiseless and the edges are sharply reproduced. From the point of view of the image quality, the results given by our method and the FAB anisotropic diffusion are comparable. However, our method requires the choice of a smaller number of parameters. This is a key advantage of the proposed approach. In order to appraise the nonlinear behavior of the different sharpeners, the luminance values of a row are graphically depicted in Figure 5. The original noise-free row number 275 (from top to bottom) is shown in Figure 5a. The corresponding row in the noisy picture is represented in Figure 5b. The significant noise increase yielded by linear UM is highlighted in Figure 5c. As above-mentioned, a smaller noise increase is produced by the nonlinear UM scheme based on the WM (Figure $5 \mathrm{~d}$ ). The result given by the PWM sharpener is shown in Figure 5e. According to our previous observation, the processed data remains as noisy as the input data, and no noise amplification is produced. The data processed by FAB anisotropic diffusion and by our method are depicted in Figures $5 \mathrm{f}$ and $5 \mathrm{~g}$, respectively. We can easily notice that, unlike the other techniques, the noise has been reduced (for a comparison, look at the noise-free data in Figure 5a).

A quantitative evaluation of the sensitivity to noise of the different sharpeners can be obtained by resorting to the mean square error (MSE) of the processed data with respect to the original uncorrupted image. Since these enhancement techniques tend to sharpen the image details, we performed the MSE evaluation by excluding the detailed areas of the image. For this purpose, we generated a map of the uniform regions by using the Sobel operator and a simple thresholding technique (threshold level = 70) [19]. The MSE values corresponding to the uniform areas of the image are reported in Table 1. We can observe that the proposed method gives the smallest MSE value. In order to evaluate the robustness of the enhancement systems, we performed a second group of tests dealing with a different noise distribution. In this experiment, we generated the noisy data by corrupting the "Tiffany" picture with uniform noise having a maximum amplitude of 16 . The different sharpening techniques were applied with no change in the parameter settings. The list of MSE values is reported in Table 2. Finally, we compared the performance of the different methods when the input image is blurred and noisy. We blurred the original "Tiffany" picture by using the $3 \times 3$ arithmetic mean filter and we added zero-mean Gaussian noise with variance 50 . 


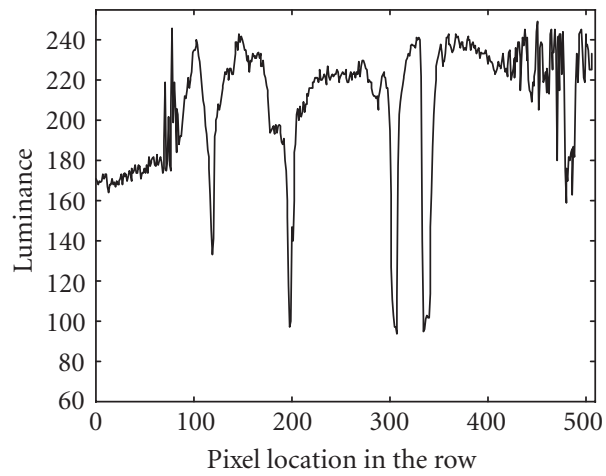

(a)

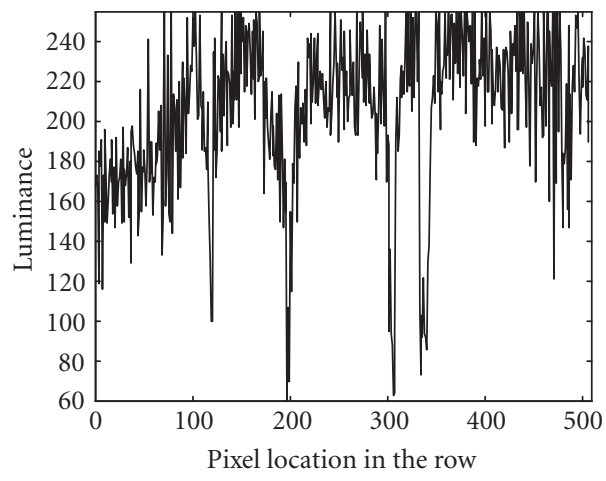

(c)

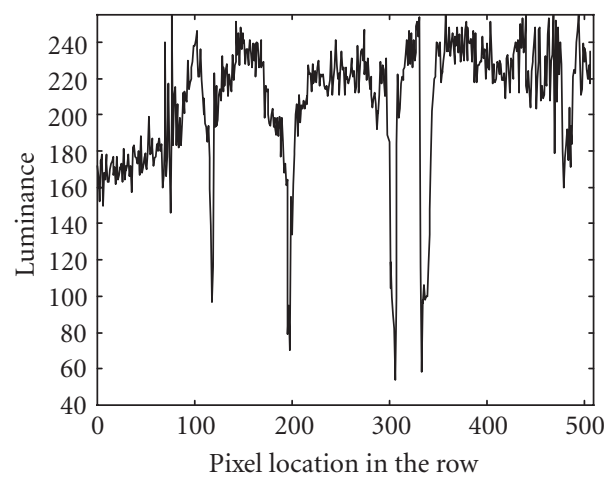

(e)

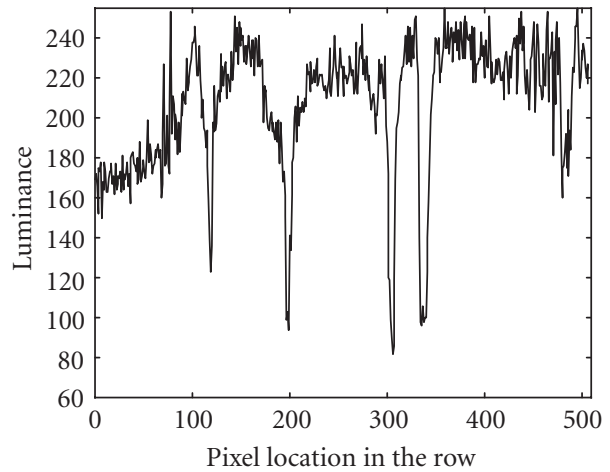

(b)

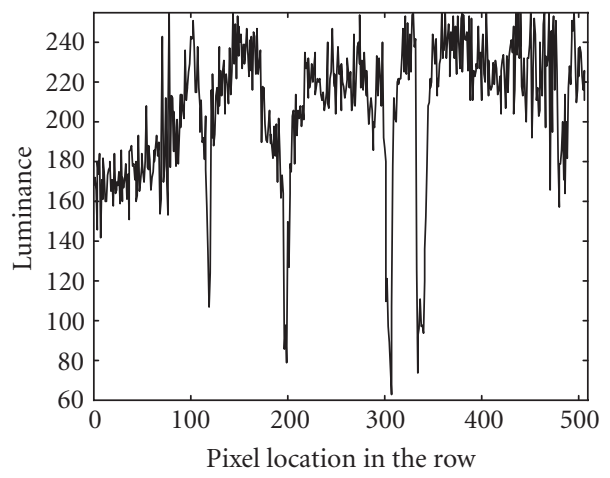

(d)

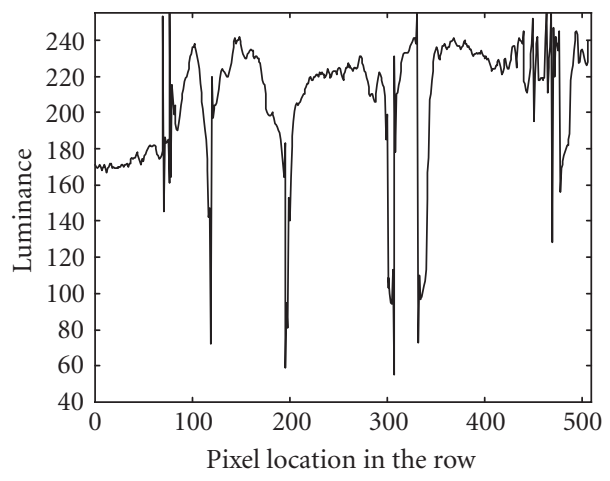

(f)

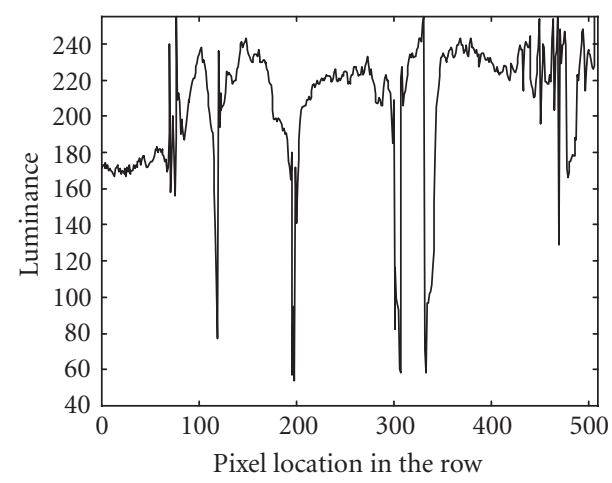

(g)

FIGURE 5: Luminance values of the row 275 (a) in the original image, (b) in the noisy image, and in the results given by (c) linear UM, (d) WM-UM, (e) PWM-UM, (f) FAB anisotropic diffusion, and (g) proposed method. 
TABLE 1: MSE values (Gaussian noise).

\begin{tabular}{l|r}
\hline IMAGE & MSE \\
\hline Corrupted by Gaussian noise & 48.6 \\
Processed: linear UM & 351.1 \\
Processed: WM-UM & 104.3 \\
Processed: PWM-UM & 50.1 \\
Processed: FAB anisotropic diffusion & 21.8 \\
Processed: proposed method & 19.9 \\
\hline
\end{tabular}

TABLE 2: MSE values (uniform noise).

\begin{tabular}{l|r}
\hline IMAGE & MSE \\
\hline Corrupted by uniform noise & 79.7 \\
Processed: linear UM & 213.1 \\
Processed: WM-UM & 105.7 \\
Processed: PWM-UM & 80.3 \\
Processed: FAB anisotropic diffusion & 76.6 \\
Processed: proposed method & 75.9 \\
\hline
\end{tabular}

TABLE 3: PSNR values.

\begin{tabular}{l|c}
\hline IMAGE & PSNR $(\mathrm{dB})$ \\
\hline Blurred and noisy & 29.2 \\
Processed: linear UM & 22.6 \\
Processed: WM-UM & 27.0 \\
Processed: PWM-UM & 30.1 \\
Processed: FAB anisotropic diffusion & 31.7 \\
Processed: proposed method & 31.8 \\
\hline
\end{tabular}

We measured the performance by using the peak signal-tonoise ratio (PSNR), which is defined as follows:

$$
\mathrm{PSNR}=10 \log _{10}\left(\frac{\sum_{\mathbf{n}} 255^{2}}{\sum_{\mathbf{n}}(y(\mathbf{n})-v(\mathbf{n}))^{2}}\right),
$$

where $v(\mathbf{n})$ denotes the luminance value of the original image at pixel location $\mathbf{n}=\left[n_{1}, n_{2}\right]$. The list of PSNR values given by the different methods is reported in Table 3. The good performance of our simple technique is apparent. The two-pass algorithm is written in C language. Look-up tables (LUTs) are currently adopted for implementing the PWL functions in order to speed up the processing. As a result, the algorithm typically requires 25 milliseconds to process a $256 \times 256 \mathrm{im}$ age on a $2.6 \mathrm{GHz}$ Pentium IV-based PC.

\section{PARAMETER TUNING}

As above-mentioned, the key feature of our technique is the combination of effectiveness and simplicity. Indeed, the choice of parameter values $k_{\mathrm{sh}}$ and $k_{\mathrm{sm}}$ is a very easy process because the nonlinear behavior is not very sensitive to them. A heuristic procedure starts by choosing a suitable value of $k_{\mathrm{sh}}$ (typically $4 \leq k_{\mathrm{sh}} \leq 6$ ) and operates by varying $k_{\mathrm{sm}}$ from zero to a value that yields a compromise between noise reduction and detail preservation. We consider some application examples.

For the sake of simplicity, let the input image be an original (noise-free) picture as depicted in Figure 6a. The activation of sharpening only $\left(k_{\mathrm{sh}}=5, k_{\mathrm{sm}}=0\right)$ produces some noise increase (Figure $6 \mathrm{~b}$ ). This effect can be corrected by activating the smoothing action $\left(k_{\mathrm{sh}}=5, k_{\mathrm{sm}}=5\right)$ as shown in Figure $6 \mathrm{c}$. The choice of $k_{\mathrm{sh}}$ is not critical. If we choose $k_{\mathrm{sh}}=$ 6 , the sharpening increase is limited (Figure 6d). Clearly, larger values of $k_{\mathrm{sh}}$ produce a stronger sharpening effect that can become annoying as shown in Figure 6e $\left(k_{\mathrm{sh}}=10\right.$, $\left.k_{\mathrm{sm}}=5\right)$ and Figure $6 \mathrm{f}\left(k_{\mathrm{sh}}=15, k_{\mathrm{sm}}=5\right)$.

The different case of a blurred image is examined in Figure 7a. A very small increase of the noise is perceivable after sharpening with $k_{\mathrm{sh}}=5$ and $k_{\mathrm{sm}}=0$ (Figure $7 \mathrm{~b}$ ). Thus, a very limited smoothing suffices to correct this effect, as depicted in Figure $7 \mathrm{c}\left(k_{\mathrm{sh}}=5, k_{\mathrm{sm}}=1\right)$. A small increase of $k_{\mathrm{sh}}$ is not critical (Figure 7d: $k_{\mathrm{sh}}=6, k_{\mathrm{sm}}=1$ ). Of course, larger values of $k_{\mathrm{sh}}$ increase the sharpening action, as represented in Figure $7 \mathrm{e}\left(k_{\mathrm{sh}}=10, k_{\mathrm{sm}}=1\right)$ and Figure $7 \mathrm{f}\left(k_{\mathrm{sh}}=15\right.$, $\left.k_{\mathrm{sm}}=1\right)$.

Finally, we consider a noisy input image. Figure 8a shows a detail of the picture represented in Figure 4b. In this case, a strong noise increase is produced after the enhancement with $k_{\mathrm{sh}}=5$ and $k_{\mathrm{sm}}=0$ (Figure $8 \mathrm{~b}$ ). Small values of $k_{\mathrm{sm}}$ do not suffice to correct this effect as shown in Figure 8c $\left(k_{\mathrm{sh}}=5, k_{\mathrm{sm}}=5\right)$ and Figure $8 \mathrm{~d}\left(k_{\mathrm{sh}}=5, k_{\mathrm{sm}}=10\right)$. A more effective smoothing is necessary in order to reduce the noise, as depicted in Figure $8 \mathrm{e}\left(k_{\mathrm{sh}}=5, k_{\mathrm{sm}}=15\right)$. Of course, too large values of $k_{\mathrm{sm}}$ produce an excess of smoothing that yields some detail blur (Figure 8f: $k_{\mathrm{sh}}=5, k_{\mathrm{sm}}=20$ ). This behavior can be taken into account in order to choose the set of optimal parameters for different types of pictures. If the image is rich in very fine details, small values of $k_{\mathrm{sm}}$ can represent a suitable choice. Conversely, if the picture is mainly composed of uniform regions, where the presence of noise is more annoying, the adoption of (slightly) larger values of $k_{\mathrm{sm}}$ can provide a better smoothing effect. In this case, the value of $k_{\mathrm{sh}}$ can be reduced in order to avoid an excess of noise increase. In this respect, an interesting improvement would be the development of an adaptive processing approach, where different parameter values are used for different pixels depending on local features. An adaptive method based on the edge gradient of the image could increase the value of $k_{\mathrm{sm}}$ in the uniform regions and then perform a stronger noise cancellation. On the contrary, smaller values of $k_{\mathrm{sm}}$ (and, possibly, larger values of $k_{\mathrm{sh}}$ ) could be adopted in presence of image details in order to improve the sharpening effect. Such an approach, where $k_{\mathrm{sh}}$ and $k_{\mathrm{sm}}$ depend on the edge gradient of the image, is a subject of present investigation.

\section{APPLICATION TO COLOR IMAGES}

When the amount of noise corruption is limited, the application of our method to noisy color images is straightforward 


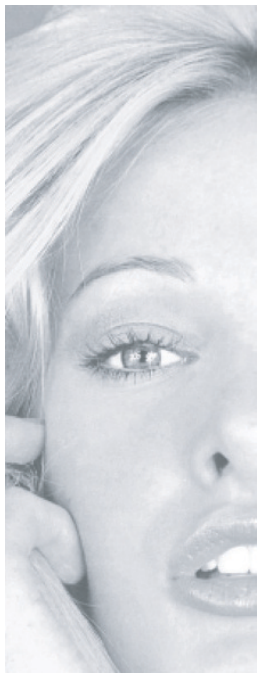

(a)

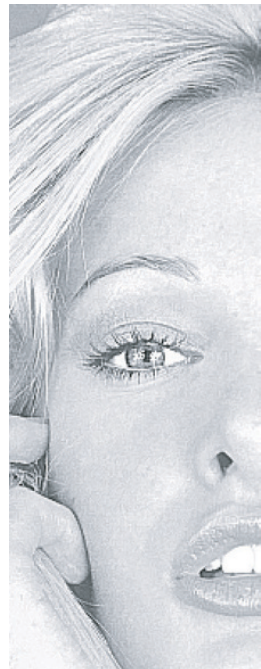

(b)

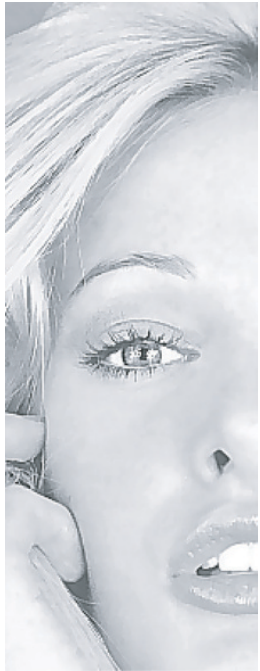

(c)

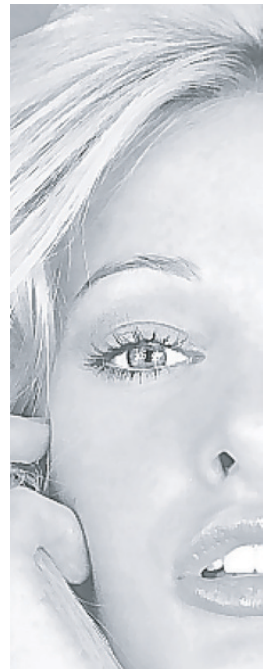

(d)

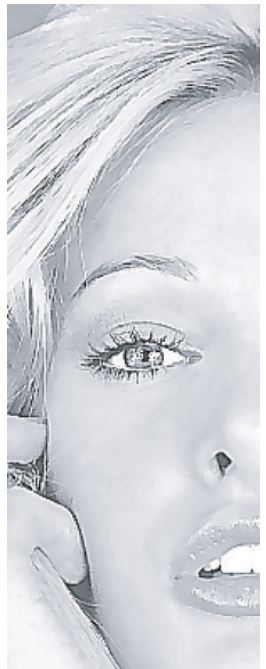

(e)

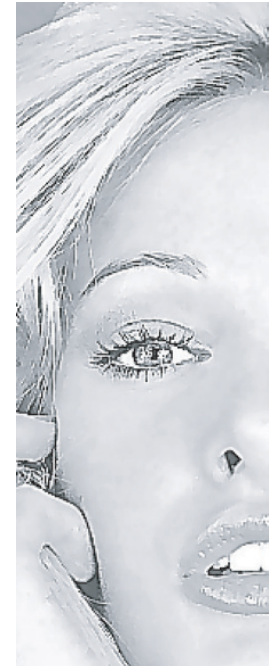

(f)

FIGURE 6: (a) Detail of original noise-free image, and results given by different parameter settings: (b) $k_{\mathrm{sh}}=5$ and $k_{\mathrm{sm}}=0,(\mathrm{c}) k_{\mathrm{sh}}=5$ and $k_{\mathrm{sm}}=5$, (d) $k_{\mathrm{sh}}=6$ and $k_{\mathrm{sm}}=5$, (e) $k_{\mathrm{sh}}=10$ and $k_{\mathrm{sm}}=5$, and (f) $k_{\mathrm{sh}}=15$ and $k_{\mathrm{sm}}=5$.

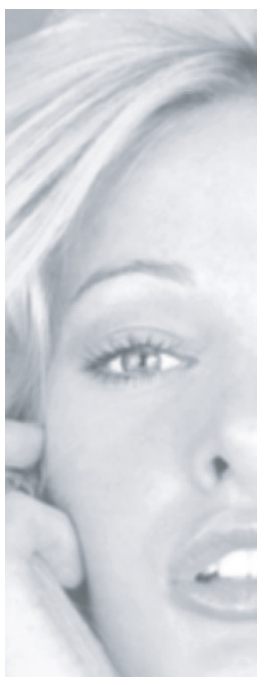

(a)

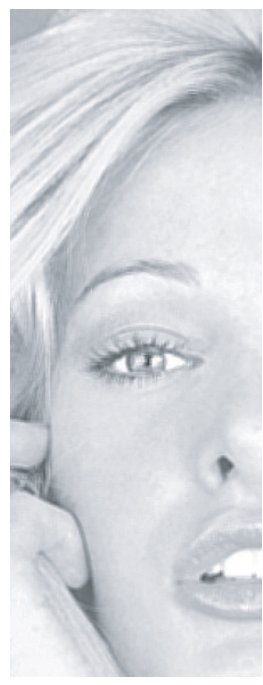

(b)

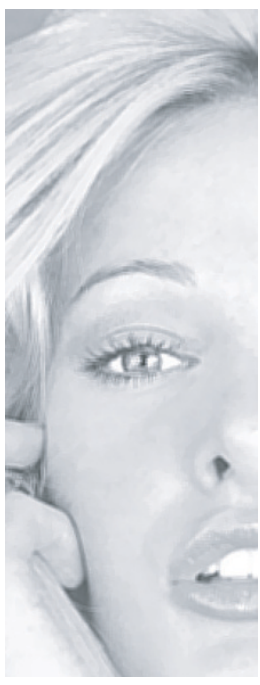

(c)

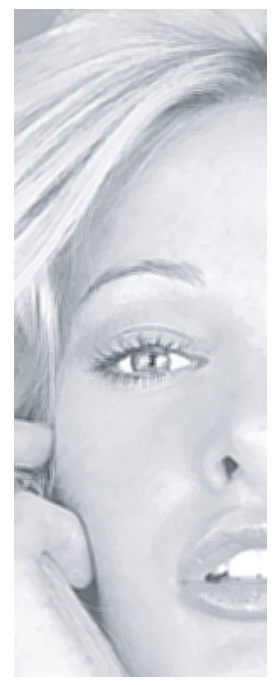

(d)

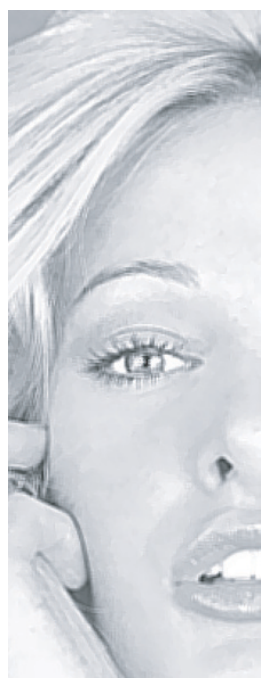

(e)

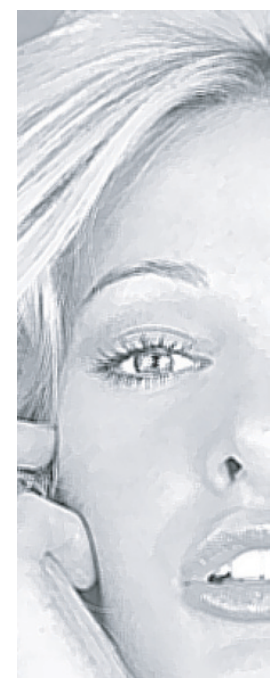

(f)

Figure 7: (a) Detail of blurred image, and results given by different parameter settings: (b) $k_{\mathrm{sh}}=5$ and $k_{\mathrm{sm}}=0$, (c) $k_{\mathrm{sh}}=5$ and $k_{\mathrm{sm}}=1$, (d) $k_{\mathrm{sh}}=6$ and $k_{\mathrm{sm}}=1$, (e) $k_{\mathrm{sh}}=10$ and $k_{\mathrm{sm}}=1$, and (f) $k_{\mathrm{sh}}=15$ and $k_{\mathrm{sm}}=1$.

and consists in processing just the luminance component. An example is reported in Figure 9. We considered the original 24-bit color picture "Tiffany" and we generated a noisy image by adding zero-mean Gaussian noise with variance 50 to the R, G, and B components (Figure 9a). Then, we adopted the YIQ color space representation [20] and we processed the luminance $\mathrm{Y}$ component only. The resulting image given by our method is shown in Figure $9 b$.

\section{CONCLUDING REMARKS}

A new nonlinear technique for contrast enhancement of noisy images has been presented. Key aspects of the proposed approach are simplicity and effectiveness. Indeed, the sharpening and smoothing actions are combined by adopting a simple PWL function whose behavior is easily controlled by two parameters only. As a result, a satisfactory compro- 


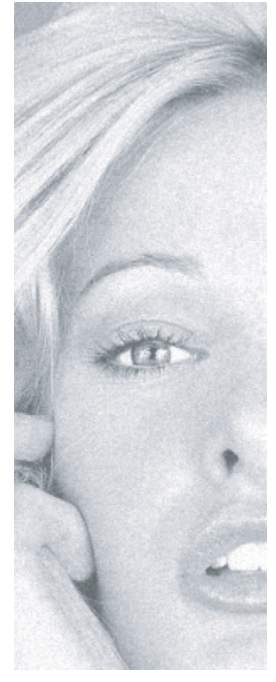

(a)

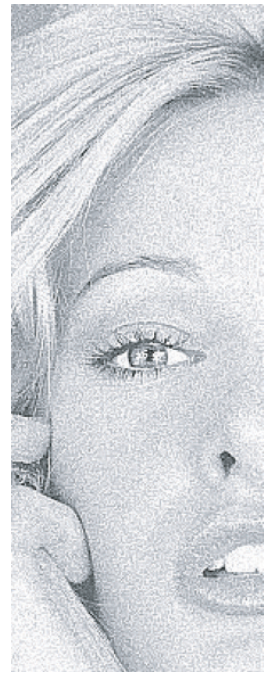

(b)

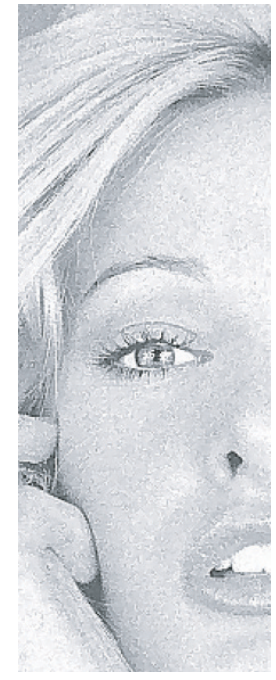

(c)

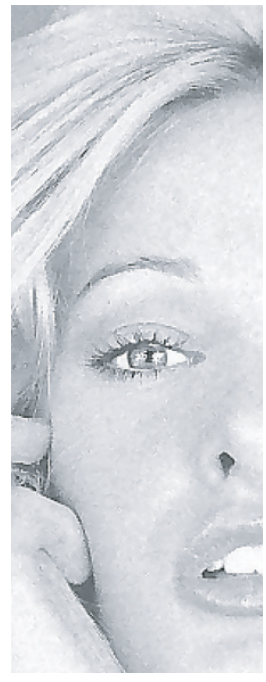

(d)

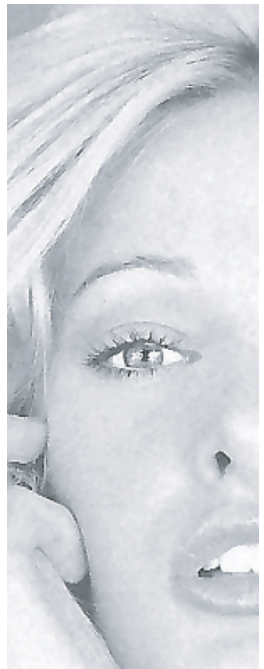

(e)

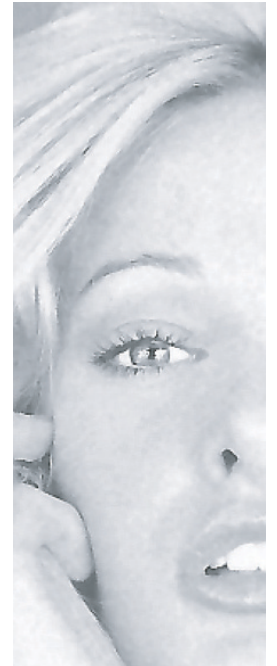

(f)

FIGURE 8: (a) Detail of noisy image, and results given by different parameter settings: (b) $k_{\mathrm{sh}}=5$ and $k_{\mathrm{sm}}=0$, (c) $k_{\mathrm{sh}}=5$ and $k_{\mathrm{sm}}=5$, (d) $k_{\mathrm{sh}}=5$ and $k_{\mathrm{sm}}=10$, (e) $k_{\mathrm{sh}}=5$ and $k_{\mathrm{sm}}=15$, and (f) $k_{\mathrm{sh}}=5$ and $k_{\mathrm{sm}}=20$.

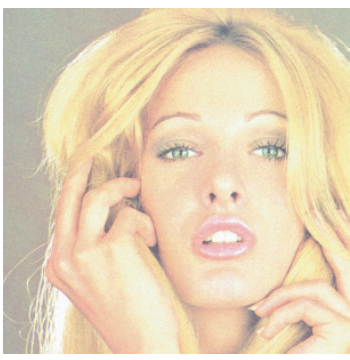

(a)

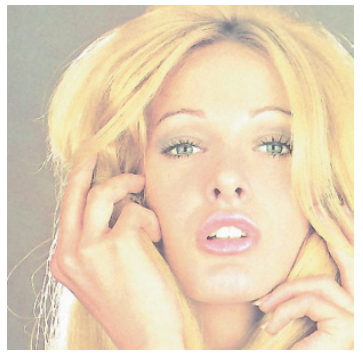

(b)
FIGURE 9: (a) Noisy 24-bit color image and (b) result of the application of the proposed method.

mise between detail sharpening and noise cancellation can be achieved. The quality of the enhanced data is improved by adopting a preprocessing step that avoids sharpening of possible outliers. The nonlinear behavior of this smoothing process is based on a different PWL model that performs a complementary action with respect to the other one.

Computer simulations have shown that the method yields very satisfactory results and that the parameter tuning is a very easy process. The method is also computationally light. As a result, potential applications to digital cameras, videocameras, and video cellular telephones can be devised.

\section{ACKNOWLEDGMENTS}

This work was supported by the University of Trieste, Italy. The source of the original images (Figures 4 and 9) is the USC-SIPI Image Database (Signal and Image Processing Institute, University of Southern California).

\section{REFERENCES}

[1] A. K. Jain, Fundamentals of Digital Image Processing, PrenticeHall, Englewood Cliffs, NJ, USA, 1989.

[2] G. Ramponi, "Polynomial and rational operators for image processing and analysis," in Nonlinear Image Processing, S. K. Mitra and G. Sicuranza, Eds., pp. 203-223, Academic Press, San Diego, Calif, USA, 2001.

[3] G. R. Arce and J. L. Paredes, "Image enhancement and analysis with weighted medians," in Nonlinear Image Processing, S. K. Mitra and G. Sicuranza, Eds., pp. 27-67, Academic Press, San Diego, Calif, USA, 2001.

[4] S. C. Matz and R. J. P. de Figueiredo, "A nonlinear technique for image contrast enhancement and sharpening," in Proc. IEEE Int. Symp. Circuits and Systems (ISCAS '99), vol. 4, pp. 175-178, Orlando, Fla, USA, May-June 1999.

[5] R. J. P. de Figueiredo and S. C. Matz, "Exponential nonlinear Volterra filters for contrast sharpening in noisy images," in Proc. IEEE Int. Conf. Acoustics, Speech, Signal Processing (ICASSP '96), vol. 4, pp. 2263-2266, Atlanta, Ga, USA, May 1996.

[6] A. Polesel, G. Ramponi, and V. J. Mathews, "Image enhancement via adaptive unsharp masking," IEEE Trans. Image Processing, vol. 9, no. 3, pp. 505-510, 2000.

[7] M. Fischer, J. L. Paredes, and G. R. Arce, "Weighted median image sharpeners for the World Wide Web," IEEE Trans. Image Processing, vol. 11, no. 7, pp. 717-727, 2002.

[8] R. C. Hardie and K. E. Barner, "Extended permutation filters and their application to edge enhancement," IEEE Trans. Image Processing, vol. 5, no. 6, pp. 855-867, 1996.

[9] J. L. Paredes, M. Fisher, and G. R. Arce, "Image sharpening using permutation weighted median filters," in Proc. European Signal Processing Conference (EUSIPCO '00), Tampere, Finland, September 2000.

[10] S. K. Mitra, H. Li, I.-S. Lin, and T.-H. Yu, "A new class of nonlinear filters for image enhancement," in Proc. IEEE Int. Conf. Acoustics, Speech, Signal Processing (ICASSP '91), vol. 4, pp. 2525-2528, Toronto, Ontario, Canada, April 1991.

[11] S. Thurnhofer, "Two-dimensional Teager filters," in Nonlinear 
Image Processing, S. K. Mitra and G. Sicuranza, Eds., pp. 167202, Academic Press, San Diego, Calif, USA, 2001.

[12] G. Ramponi, N. Strobel, S. K. Mitra, and T.-H. Yu, "Nonlinear unsharp masking methods for image contrast enhancement," Journal of Electronic Imaging, vol. 5, no. 3, pp. 353-366, 1996.

[13] G. Ramponi and A. Polesel, "Rational unsharp masking technique," Journal of Electronic Imaging, vol. 7, no. 2, pp. 333338, 1998.

[14] F. Russo and G. Ramponi, "Fuzzy operator for sharpening of noisy images," Electronics Letters, vol. 28, no. 18, pp. 17151717, 1992.

[15] F. Russo and G. Ramponi, "Nonlinear fuzzy operators for image processing," Signal Processing, vol. 38, no. 3, pp. 429-440, 1994.

[16] F. Russo and G. Ramponi, "An image enhancement technique based on the FIRE operator," in Proc. IEEE International Conference on Image Processing (ICIP '95), vol. 1, pp. 155-158, Washington, DC, USA, October 1995.

[17] M. Black, G. Sapiro, D. Marimont, and D. Heeger, "Robust anisotropic diffusion and sharpening of scalar and vector images," in Proc. IEEE International Conference on Image Processing (ICIP '97), vol. 1, pp. 263-266, Santa Barbara, Calif, USA, October 1997.

[18] B. Smolka, M. Szczepanski, K. N. Plataniotis, and A. N. Venetsanopoulos, "Forward and backward anisotropic diffusion filtering for color image enhancement," in Proc. 14th IEEE International Conference on Digital Signal Processing (DSP '02), vol. 2, pp. 927-930, Santorini, Greece, July 2002.

[19] F. Russo, "An image enhancement technique combining sharpening and noise reduction," IEEE Trans. Instrumentation and Measurement, vol. 51, no. 4, pp. 824-828, 2002.

[20] I. Pitas, Digital Image Processing Algorithms and Applications, John Wiley \& Sons, New York, NY, USA, 2000.

Fabrizio Russo obtained the Dr.-Ing. degree in electronic engineering (with the highest honors) in 1981 from the University of Trieste, Trieste, Italy. In 1984, he joined the Department of Electrical, Electronic and Computer Engineering (DEEI) of the University of Trieste, where he is currently an Associate Professor of electrical and electronic measurements. His main interests are in the field of nonlinear signal processing

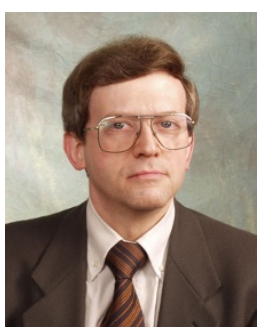
based on computational intelligence for instrumentation and measurement. His research activity focuses on nonlinear models for image enhancement and edge detection, fuzzy and neurofuzzy filters for noise cancellation, techniques for objective evaluation of image quality, and intelligent instrumentation. His research results have been published in more than 80 papers in international journals, textbooks, and conference proceedings. Professor Russo is a member of the IEEE. 